\title{
NUTRIENT SHORTFALLS IN YOUNG \\ CHILDREN'S DIETS AND THE ROLE OF AFFORDABILITY
}

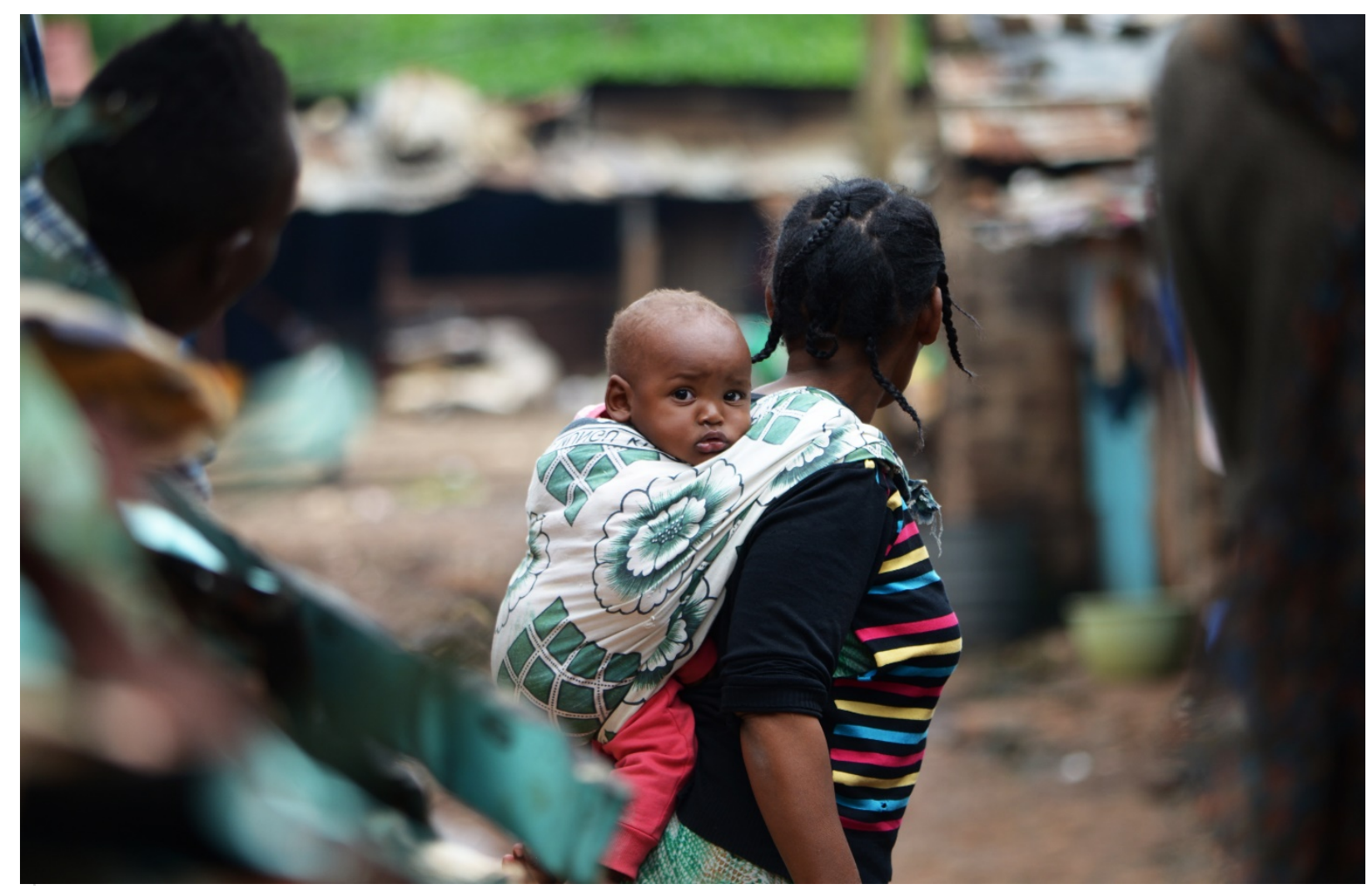

GAIN Briefing Paper ${ }^{\circ} 4$

March, 2021

Ty Beal 


\section{ABOUT GAIN}

The Global Alliance for Improved Nutrition (GAIN) is a Swiss-based foundation launched at the UN in 2002 to tackle the human suffering caused by malnutrition. Working with governments, businesses and civil society, we aim to transform food systems so that they deliver more nutritious food for all people, especially the most vulnerable.

\section{Recommended citation}

Beal, T. Nutrient shortfalls in young children's diets and the role of affordability. Global Alliance for Improved Nutrition (GAIN). Briefing Paper \#4. Geneva, Switzerland, 2021. DOI:

https://doi.org/10.36072/bp.4

\section{() The Global Alliance for Improved Nutrition (GAIN)}

This work is available under the Creative Commons Attribution-Non-Commercial-Share Alike 4.0 IGO licence (CC BY-NC-SA 4.0 IGO; https://creativecommons.org/licenses/by-nc-sa/4.0/). Under the terms of this licence, you may copy, redistribute and adapt the work for non-commercial purposes, provided the work is appropriately cited, as indicated below. In any use of this work, there should be no suggestion that GAIN endorses any specific organisation, products or services. The use of the GAIN logo is not permitted. If you adapt the work, then you must license your work under the same or equivalent Creative Commons license. The contribution of third parties do not necessarily represent the view or opinion of GAIN.

\section{Acknowledgements}

I thank Jessica Fanzo, Lynnette Neufeld, Heidi Fritschel, Theresa Ryckman, Jessica White, Stella Nordhagen, Aashima Garg, Shahira Malm, and Saul Morris for their helpful feedback on draft versions of this manuscript, and Danielle DeGarmo for support with Graphic Design.

This paper is based on a supplement of the journal Nutrition Reviews (Volume 79, Suppl 1) available here. It also builds on a set of country-specific briefs created for Bangladesh, Ethiopia, India, Mozambique, Pakistan, South Africa, Tanzania, Uganda, and Zambia; the briefs on nutrient gaps are available here and those on affordable foods to fill them are available here.

All photographs included in this document have been taken with consent for use in publications.

\section{GAIN BRIEFING PAPER SERIES}

GAIN Briefing Paper Series provide essential information in a succinct, accessible form to support informed decision-making by stakeholders in the food system to improve the consumption of nutritious, safe food for all people, especially the most vulnerable.

The Global Alliance for Improved Nutrition (GAIN)

Rue de Varembé 7

1202 Geneva

Switzerland

$\mathrm{T}:$ +41 227491850

E: info@gainhealth.org

www.gainhealth.org 


\section{SUMMARY}

For millions of children in Eastern and Southern Africa and South Asia, current diets do not contain enough nutrients for proper growth and development. This is a tragedy. New evidence has recently been published that sheds light on the nutrient gaps experienced by young children in 14 countries in these regions and examines which foods might be affordably used to fill them. This briefing paper highlights the key findings from this research. In both regions, the most common vitamin and mineral shortfalls in young children's diets are in iron, vitamin $A$, zinc, folate, vitamin $B_{12}$, and calcium. Many nutritious foods containing high amounts of these nutrients are available in countries within these regions, including liver, small fish, beef or goat meat, eggs, and dark green leafy vegetables. The affordability of nutritious foods varies, with the most affordable sources being liver, small fish, dark green leafy vegetables, milk, and eggs. These results have important implications for policymakers and programme designers working to solve this urgent crisis of poor diets and undernutrition.

\section{KEY MESSAGES}

- The majority of young children in Eastern and Southern Africa and South Asia have extremely poor diets, resulting in undernutrition that is preventing proper growth and development.

- The most common vitamin and mineral gaps in young childrern's diets in the two regions are in iron, vitamin $A$, zinc, folate, vitamin $B_{12}$, and calcium.

- The best food sources of nutrients to fill these commom nutrient gaps are liver, small fish, beef or goat meat, eggs, and dark green leafy vegetables.

- Affordability is a barrier to accessing nutritious foods, particularly in quantities required to meet needs for iron and zinc.

- The most affordable sources of commonly lacking nutrients are liver, small fish, dark green leafy vegetables, milk, and eggs.

- These evidence-based findings can be used to design programmes and policies, by identifying affordable foods for children that meet important nutrient gaps. 


\section{INTRODUCTION}

Millions of children across the world do not consume enough essential vitamins and minerals. These 'micronutrients' are crucial for immunity, cellular growth and protection, and a vast array of other functions critical for human health, particularly child growth and development. For children who consume inadequate amounts of these micronutrients during the complementary feeding period-the period when infants and young children are 6-23 months old and breastmilk alone is no longer sufficient to meet their needs-the short- and long-term consequences can be severe. Although shortfalls in consumption of dietary energy - that is, calories-also constitute an issue of paramount importance, shortfalls in children's consumption of vitamins and minerals are a distinct problem: inadequate micronutrient consumption may occur with or without adequate calorie consumption.

A supplement of the journal Nutrition Reviews (available here), provides evidence on nutrient gaps in young children's diets and insight into a key determinant (affordability) in countries in South Asia and Eastern and Southern Africa that suffer from high burdens of childhood malnutrition. This briefing paper summarises the key results of this supplement, highlighting those of greatest policy and programmatic relevance.

\section{IDENTIFYING SHORTFALLS IN YOUNG CHILDREN'S DIETS}

To address micronutrient shortfalls, it is helpful to know which foods to prioritise in children's diets. However, it is not always easy to identify the micronutrients lacking in the diets of a given population and pinpoint the ones that are particularly hard to get in sufficient quantities. Data on so-called nutrient gaps are not widely collected owing to financial and logistical constraints, and the existing data contain different types of information that can be challenging to interpret. Biomarkers assess micronutrient status through laboratory tests to identify shortfalls in the body, but because they generally identify issues only once they have become severe, they have limited potential to inform prevention efforts. Data on the foods households consume provide insight into potential shortfalls, but not everyone within a household eats equally, so these data may not identify individuals at risk. Risk can be better assessed using individual-level dietary intake data, but these are missing in many countries and subgroups within countries. Food supply data, such as that compiled regularly by the Food and Agriculture Organization, can point only to shortfalls in the overall food system. Each of these data sources provides some insights into the dietary nutrient gaps that can be useful for agricultural and health policy, but no one data source alone is sufficient to identify the micronutrients most likely to be deficient in young children's diets and to suggest how to address these gaps. Moreover, there remains a lack of global consensus on the public health significance of different prevalence ranges for several common micronutrient deficiencies, such as iron, zinc, folate, vitamin $B_{12}$, calcium, and vitamin D. For example, if $12 \%$ of the population is iron deficient, how important of a public health issue is that? Is there a similar or different public health burden if $12 \%$ of the population is folate deficient?

The first research paper in the above-mentioned supplement describes a new approach for examining these various data sources to understand and communicate the public health importance of nutrient gaps and related evidence gaps (1). The approach accounts for 
differences in data quality and recency as well as for disagreements between data points, and it provides ratings of the certainty of the evidence underlying them.

\section{MICRONUTRIENT GAPS AND THE FOODS THAT CAN FILL THEM}

This approach was then used to estimate the burden of micronutrient gaps in young children's diets in six countries in Eastern and Southern Africa (2) and eight countries in South Asia (3). In both regions, biomarker data are available for iron and vitamin A. In South Asia, biomarker data are available for zinc for most countries and for folate and vitamin $\mathrm{B}_{12}$ for some countries. Few biomarker data are available in either region for other nutrients, especially calcium. In the six countries studied in Eastern and Southern Africa, the available evidence consists mostly of data on nutrients in the food supply for zinc, folate, and vitamin $\mathrm{B}_{12}$. Overall, there is a lack of data on dietary nutrient adequacy. Applying the new estimation approach to the available evidence, the papers find that the most common micronutrient gaps in the two regions are in iron, vitamin $A$, zinc, folate, vitamin $B_{12}$, and calcium.

These micronutrient gaps could be addressed by improving the quality of young children's diets by incentivising the production and promoting the consumption of minimally processed nutritious foods (including biofortified foods), fortifying staple foods with micronutrients, and providing micronutrient supplements. Improving diet quality is particularly important because minimally processed foods contain essential nutrients in forms that are most appropriate for the body to metabolise. They also contain thousands of other compounds, such as antioxidants and polyphenols, that are bound together in a food matrix and seem to play an important role in health (3).

Interestingly, many foods available in the study countries contain large amounts of critical nutrients. Available foods were assessed for how well they met the needs for the six micronutrients commonly lacking in young children's diets: iron, vitamin A, zinc, folate, vitamin $B_{12}$, and calcium. Figure 1 (adapted from the data in the papers of the abovementioned journal supplement) shows three tiers of foods ranked by their levels of these six micronutrients. The bars indicate the portion size needed to provide an average of one-third of daily requirements for these six nutrients from complementary foods for a child 6-23 months old (the equivalent of $100 \%$ adequacy for two micronutrients or $33.3 \%$ adequacy for all six micronutrients). This metric demonstrates the ideal foods to fill two or more important micronutrient gaps simultaneously. Foods in tier 1, which require portion sizes of only $25 \mathrm{~g}$ or less, are considered optimal sources and include liver and small dried fish. Foods in tier 2 , 


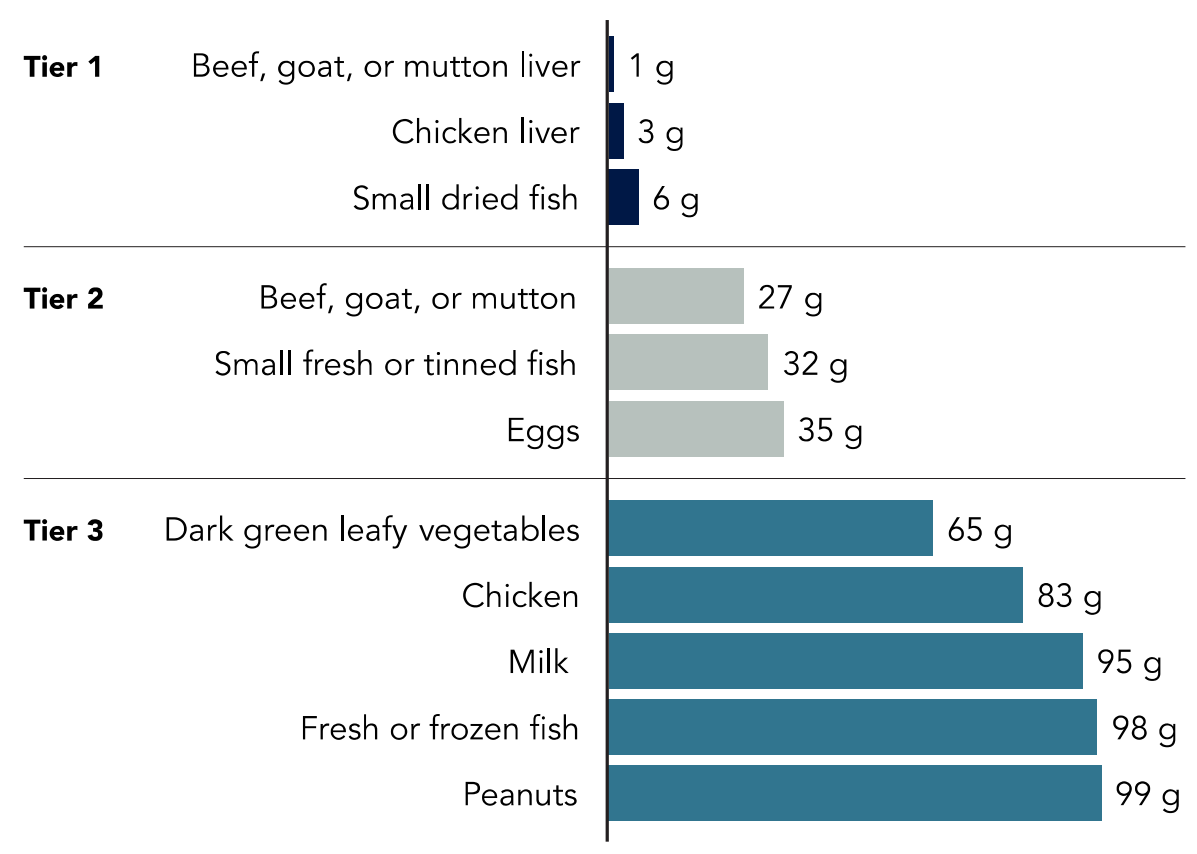

Figure 1. Portion size needed to provide an average of one-third of a young child's requirements from complementary foods for iron, vitamin $A$, zinc, folate, vitamin $B_{12}$, and calcium. The share of daily requirements of each nutrient provided by the specified quantity of food was capped at $100 \%$. Data are for children 6-23 months old. Data are from $(2,3)$.

which require portion sizes of 25-50 g, are considered adequate sources and include beef, goat, and mutton; small fresh or tinned fish; and eggs. Foods in tier 3 , which require portion sizes of 50-99 g, are considered moderately adequate sources and include dark green leafy vegetables, chicken, milk, fresh or frozen fish (excluding small fish), and peanuts. Increasing young children's consumption of foods in tiers 1, 2, and 3, with preference for higher tiers, is essential for achieving nutrient adequacy. These foods are also the most relevant foods for other groups vulnerable to undernutrition and similar micronutrient deficiencies, such as older children, adolescents, and women of reproductive age (especially pregnant and lactating women).

\section{AFFORDABILITY OF NUTRITIOUS FOODS}

After the nutritious foods to fill critical nutrient gaps have been identified, the next question is, are they affordable? Traditionally, the affordability of individual foods or food groups has been assessed on a per calorie or per kilogram basis, but when the goal is to consume foods with enough nutrients, affordability must be measured based on nutrient content. An additional contribution of this work was to develop an approach to do so, using data on household consumption and expenditure, food prices, and food composition data. This method was applied to assess the affordability of the key nutrients commonly lacking in young children's diets $(4,5)$. 


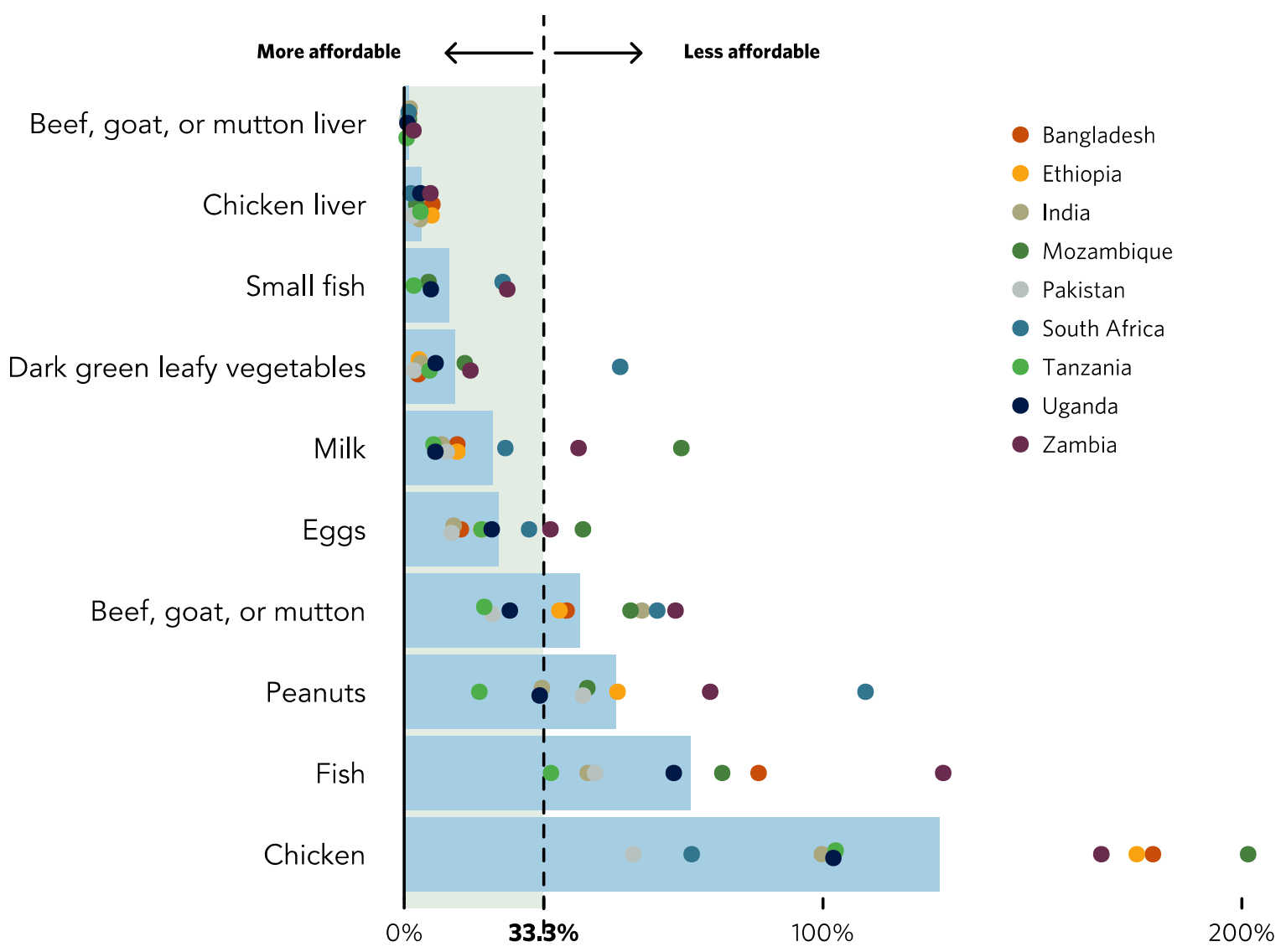

Figure 2. Share of food expenditures per person needed to provide an average of one-third of a young child's requirements for iron, vitamin $A$, zinc, folate, vitamin $B_{12}$, and calcium. Bars show averages from nine countries in Eastern and Southern Africa and South Asia from data collected between 2011 and 2016. Points show values for individual countries. The affordability threshold (dashed line) was set at one-third (33.3\%) of food expenditures because this analysis is based on meeting an average of one-third of requirements for six micronutrients from complementary foods. The share of daily requirements of each nutrient provided by the specified quantity of food was capped at 100\%. Data are for children 6-23 months old. Data are from refs $(4,5)$.

Foods were assessed in terms of their affordability for meeting needs for several micronutrients in combination. Figure 2 shows the average share of food expenditures per person needed to provide an average of one-third of a young child's requirements for iron, vitamin $A$, zinc, folate, vitamin $B_{12}$, and calcium from complementary foods. The shares of food expenditure were based on averages across six countries in Eastern and Southern Africa and three in South Asia. Bars to the left of the dotted line indicate foods that are affordable sources of two or more of the six micronutrients. Based on this aggregate metric, affordable foods in Eastern and Southern Africa and South Asia include liver, small fish, dark green leafy vegetables, milk, and eggs. In some countries beef, goat, mutton, and peanuts are affordable; in all countries fish (excluding small fish) and chicken are unaffordable. Although animal-source foods are an expensive source of calories, they can be an affordable source of micronutrients given the lower quantities required to contribute significantly to key nutrient needs. Nevertheless, most consumers, especially those with low food budgets, may not value foods primarily for their nutrient content or fully realise the importance of selecting particularly nutrient-dense foods for young children. Rather, foods are commonly valued for 
their calories, aspirational value, taste, and convenience-criteria that tend to favour highly processed foods.

The papers also assessed the affordability of foods as sources of single nutrients. For this metric, affordability varies across countries, but clear patterns emerge. First, across both regions several foods are affordable sources of vitamin $A$ and $B_{12}$, including liver for both vitamins, fish for vitamin $B_{12}$, and dark green leafy vegetables and orange-fleshed vegetables for vitamin $A$. Thus, it appears that unaffordability is not a key barrier to adequate consumption of vitamin $A$ and $B_{12}$. Second, few if any single-food sources of iron and zinc are affordable for poor households in the quantities required to meet young children's high needs. While beef, goat, and mutton; liver; and small dried fish contain high amounts iron and zinc, they are not affordable sources for many households. Even the most affordable sources (often dark green leafy vegetables for iron and pulses for zinc) are frequently unaffordable. Without substantial reductions in prices, raised incomes, social protection programmes, or home production, poor populations are unlikely to be able to consume enough iron and zinc through foods.

\section{CONCLUSION}

In summary, the new evidence contributed by this journal supplement has provided innovative methods for assessing nutrient gaps, identifying nutritious foods to fill them, and measuring the affordability of those foods. While these methods can be used for any population, to date they have been used to assess how to improve nutrition amongst young children through adequate complementary feeding in Eastern and Southern Africa and South Asia. The diets of a large share of young children in these regions are extremely poor, resulting in undernutrition that prevents proper growth and development. Unaffordability is a major barrier to consumption of many nutritious foods, particularly in the quantities required to meet needs for iron and zinc. These new insights should be used to design programmes and policies that are grounded in evidence and effective in reaching the most vulnerable children, providing them with the ability to thrive and realise their full potential. Finally, in many populations, particularly in Eastern and Southern Africa, more data are needed on micronutrient biomarkers and diets to better understand shortfalls in the diet and how to address them. 


\section{REFERENCES}

1. Beal T, White JM, Arsenault JE, Okronipa H, Hinnouho G-M, Morris SS. Comprehensive Nutrient Gap Assessment (CONGA): A method for identifying the public health significance of nutrient gaps. Nutrition Reviews. 2021;79(Suppl 1)

2. White JM, Beal T, Chimanya K, Arsenault JE, Okronipa H, Hinnouho G-M, et al. Micronutrient gaps during the complementary feeding period in Eastern and Southern Africa: A Comprehensive Nutrient Gap Assessment. Nutrition Reviews. 2021;79(Suppl 1)

3. Beal T, White JM, Arsenault JE, Okronipa H, Hinnouho G-M, Torlesse H, et al. Micronutrient gaps during the complementary feeding period in South Asia: $A$ Comprehensive Nutrient Gap Assessment. Nutrition Reviews. 2021; 79(Suppl 1)

4. Ryckman T, Beal T, Nordhagen S, Chimanya K, Matji J. Affordability of nutritious foods for complementary feeding in Eastern and Southern Africa. Nutrition Reviews. 2021;79(Suppl 1).

5. Ryckman T, Beal T, Nordhagen S, Murira Z, Torlesse H. Affordability of nutritious foods for complementary feeding in South Asia. Nutrition Reviews. 2021;79(Suppl 1). 